\title{
Exploring the neglected segment of the intestine: the duodenum and its pathologies
}

\author{
Anjuna Reghunath ${ }^{A, B, C, D, E, F}$, Kavirajan Kabilan ${ }^{B, E, F}$, Mahesh K. Mittal| ${ }^{A, D, E, F}$ \\ Department of Radiology, Vardhman Mahavir Medical College and Safdarjung Hospital, New Delhi, India
}

\begin{abstract}
Herein we reviewed the computed tomography (CT) findings of a spectrum of pathological entities affecting the duodenum. We discuss the CT findings of some congenital, inflammatory, traumatic, and neoplastic pathologies of the duodenum along with the conventional barium studies of selected conditions. Pathologies of this C-shaped intestinal segment, derived from both foregut and midgut, are often overlooked in clinical practice and radiological literature. While congenital anomalies like duplication cysts and diverticula are usually asymptomatic, annular pancreas and malrotation may manifest in the first decade of life. Primary as well as secondary involvement of the duodenum by various disease processes can be evaluated by careful CT technique and proper attention to the duodenum. Among congenital conditions, annular pancreas, duplication cyst, superior mesenteric artery syndrome, midgut volvulus, and diverticula are presented. Duodenal involvement in adenocarcinoma, lymphoma, gastrointestinal stromal tumours, Crohn's disease, and groove pancreatitis are discussed. Duodenal wall haematoma and traumatic duodenal perforation causing pneumoretroperitoneum in two patients after blunt trauma of the abdomen are also illustrated. CT provides superb anatomic detail and offers high diagnostic specificity for the detection of duodenal pathologies because it allows direct imaging of the intestinal wall, secondary signs of bowel disease within the surrounding mesentery, and abnormal findings in adjacent structures. Primary duodenal malignancies and local extension from adjacent malignancies can be diagnosed by CT reliably. CT also plays a vital role in the diagnosis of traumatic duodenal injury by differentiating between mural haematoma and a duodenal perforation because the latter requires immediate surgical intervention.
\end{abstract}

Key words: computed tomography, duodenum, abdominal imaging.

\section{Introduction}

Pathologies of the duodenum are often overlooked in clinical practice and radiological literature. Its location, development, and proximity to intraperitoneal organs such as liver, gall bladder, stomach, and retroperitoneal structures like pancreas, aorta, spine, and ascending colon result in duodenal involvement by a multitude of primary and secondary processes. These processes can be well evaluated by careful computed tomography $(\mathrm{CT})$ technique and proper attention to the duodenum. This review aims to explore the CT findings of the spectrum of pathological entities affecting the duodenum along with a simplified radiological approach, and to discuss the recent advances in imaging of the duodenum.

\section{Development of duodenum}

The duodenum is a C-shaped hollow viscus of approx. length $20-30 \mathrm{~cm}$, with unique developmental attributes. It is partly intraperitoneal and partly extraperitoneal, takes its origin from both foregut and midgut, and is incompletely covered by peritoneum without any mesentery. Physiological umbilical herniation of intestine occurs in the sixth week of intrauterine life [1]. The primary intestinal loop turns 270 degrees anticlockwise around an axis formed by the superior mesenteric artery. The herniated loops of intestine return back to the abdominal cavity at the $10^{\text {th }}$ week of intrauterine life. The proximal jejunal portion and caecal bud are the first and last part of the gut to return into the abdominal cav-

\section{Correspondence address:}

Prof. Mahesh Kumar Mittal, Department of Radiology, Vardhman Mahavir Medical College and Safdarjung Hospital, India, e-mail: drmittalmk@gmail.com

Authors' contribution:

A Study design · B Data collection · C Statistical analysis · D Data interpretation · E Manuscript preparation · F Literature search · G Funds collection 
ity and occupy the left and right sides of the abdominal cavity, respectively.

The duodenum consists of four segments [1]:

- First part/superior part - the sole intraperitoneal segment is the first $2 \mathrm{~cm}$ of the proximal duodenum, called the duodenal cap or bulb. The gastroduodenal artery and portal vein run posterior to the first part.

- Second part/descending part - it extends from the superior duodenal flexure at the L1 vertebra to the inferior duodenal flexure at the L3 vertebra. The second part of the duodenum contains major and minor duodenal papilla. The main pancreatic duct (Wirsung duct) and common bile duct open into the major duodenal papilla while the accessory pancreatic duct (duct of Santorini) opens into the minor duodenal papilla. The embryological intersection between the foregut and hindgut lies immediately below the major duodenal papilla.

- Third part/horizontal part - it crosses from right to left and it is related anteriorly to the superior mesenteric vessels and posteriorly to the abdominal aorta and interior vena cava.

- Fourth part/ascending part - it is located left of the abdominal aorta over the left psoas muscle and passes upwards to the level of the L2 vertebra and ends at the duodenojejunal flexure.

\section{Computed tomography technique}

Routine CT scans of the abdomen are procured after the administration of oral contrast material for adequate distension and visualisation of the gastrointestinal tract. One technique used to optimise duodenal imaging is the use of bicarbonate granules to expand the stomach and duodenum [1]. In suspected mucosal pathologies,

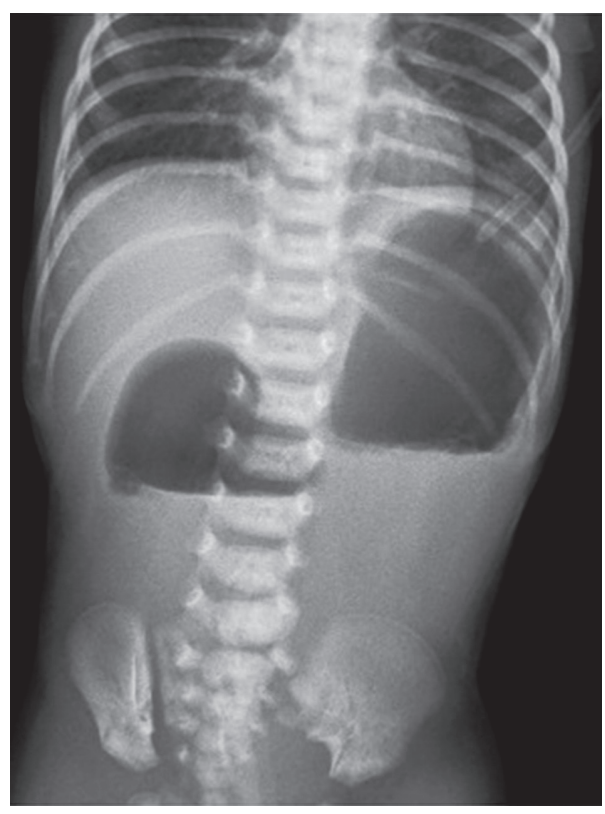

Figure 1. Frontal radiograph of the abdomen and lower thorax showing the typical 'double bubble' sign seen in a case of congenital duodenal atresia negative contrast agents, like water or mannitol, can be given peroral for superb contrast with the duodenal wall. The patient is placed in the right posterior oblique position to achieve the best opacification of first and second duodenal segments, and in the left posterior oblique position to distend the duodenum when carbon dioxide is used. Intravenous iodinated contrast material is injected at the rate of $4-5 \mathrm{ml} / \mathrm{s}$ for delineating enhancement pattern of lesions within and adjacent to the duodenum. Conventionally, a pitch of 1.0 with $5 \mathrm{~mm}$ collimation is usually chosen, with thinner-collimation images $(<3.0 \mathrm{~mm})$ being reconstructed for better anatomic resolution. Multiplanar reformats in oblique and coronal planes of the duodenum may be done [1].

\section{Pathologies of the duodenum}

We discuss the CT findings of some congenital, infective, inflammatory, traumatic, vascular, and neoplastic pathologies of duodenum along with the conventional barium studies of selected conditions.

\section{Congenital anomalies}

\section{Duodenal atresia}

It is the commonest congenital cause of duodenal obstruction, occurring due to failed recanalisation between 6 and 11 weeks. The total failure of recanalisation results in atresia, while partial failure results in stenosis [2]. Duodenal atresia shows a typical 'double bubble' sign and the absence of small and large bowel gas on plain radiograph (Figure 1). It is usually post ampullary in $85 \%$ of cases [3], and patients present with bilious vomiting within two days of birth. Duodenal web is a type 1 duodenal atresia in which a thin diaphragm-like membrane forms in the duodenal lumen secondary to defective recanalisation. High pressure in the proximal duodenum causes the web to bulge into the distal duodenum, thereby producing characteristic 'windsock deformity'.

\section{Annular pancreas}

The occurrence of pancreatic tissue posterolateral to the descending duodenum is specific for diagnosing annular pancreas [4]. A complete loop of pancreatic tissue is not always required for diagnosis. Triangular pancreatic tissue surrounding the descending duodenum giving the characteristic crocodile jaw configuration is seen in incomplete annular pancreas (Figure 2). Portal annular pancreas is an infrequent anomaly and is allied with an increased incidence of postoperative pancreatic fistula following pancreatic resection. Demonstration of 'pancreatic duct ring' sign on ERCP/MRCP is diagnostic for portal annular pancreas [5]. Post-ampullary duodenal obstruction, pancreas divisum (Figure 3), pancreatitis, and pancreatobili- 


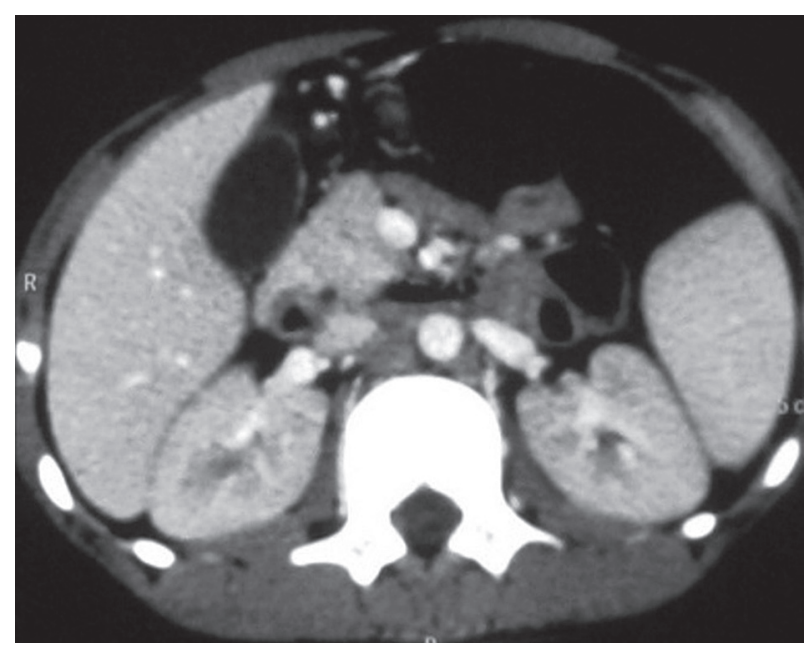

Figure 2. Axial sections of contrast-enhanced computed tomography abdomen showing partial encircling of the duodenum by pancreatic parenchyma giving the characteristic crocodile-jaw configuration, suggestive of incomplete annular pancreas

ary neoplasia are common in adults [6]. Plain radiograph often demonstrates the classic 'double bubble' sign. Focal constriction of the second segment of the duodenum can be demonstrated in barium meal examination.

\section{Duodenal diverticula}

Most true diverticulae are situated in the medial wall of the second part of the duodenum in the periampullary region and appear as smooth outpouchings (Figure 4). Giant duodenal diverticula usually arise in the lateral aspect. Bizarre multilobulated (racemose) diverticula can also occur. On barium study, they appear as round, smooth outpouchings without any surrounding signs of inflammation or change in shape during the course of study. The complications of duodenal diverticulae are diverticulitis, perforation, upper GI bleeding and pancreaticobiliary disease [1]. When duodenal diverticulum in periampullary location compresses the intrapancreatic part of the common bile duct (CBD) with resultant upstream dilatation of extra- and intrahepatic bile ducts, leading to obstructive jaundice; the condition is called Lemmel syndrome (Figure 4).

Differential diagnoses of duodenal diverticula are:

- Giant duodenal ulcer - its shape remains constant throughout the study, lacks normal mucosal folds, and inflammatory reaction surrounding the ulcer causes contraction of the circular muscle, producing incisure just opposite to the ulcer.

- Pseudodiverticula - usually secondary to peptic ulcer disease and seen as sacculations in the superior and inferior recesses of the duodenal bulb.

- Intraluminal diverticula - barium filled sac seen intraluminally, which is separated from the adjacent duodenal wall by a radiolucent line and seen as windsock deformity in the second part of the duodenum.
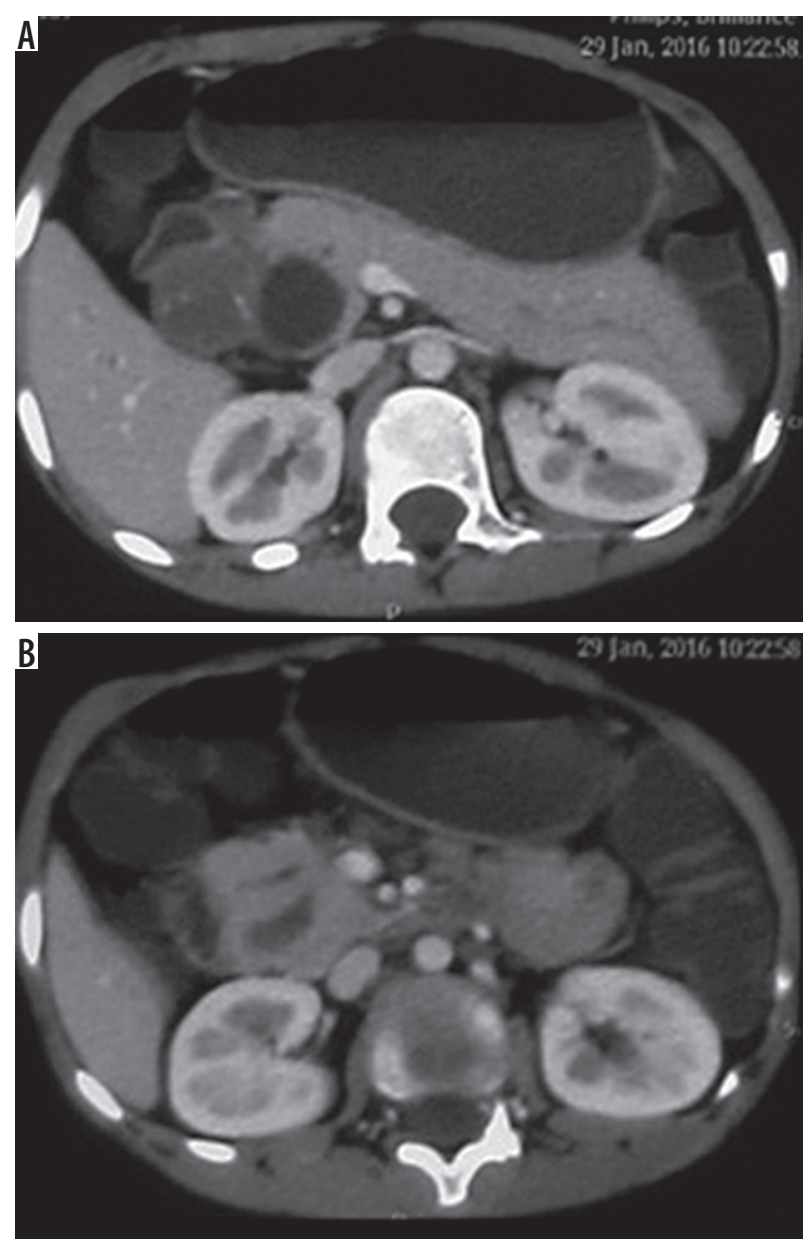

Figure 3. Axial contrast-enhanced computed tomography sections showing separate dorsal and ventral pancreatic duct just before insertion into the duodenum, suggesting pancreas divisum

\section{Duodenal duplication cyst}

The most common type of duplication cyst is cystic and non-communicating [7]. The muscular layer of the medial aspect of the first and second parts of the duodenum is the most frequent location of duodenal duplication cyst. Although mostly asymptomatic, patients may present with abdominal pain, vomiting, pancreatitis, and obstructive jaundice. It rarely causes complete duodenal obstruction [8]. Although duodenal duplication is benign, malignancies arising from the cyst are also reported. On barium study, it is seen as a well-defined, smooth, extrinsic filling defect projecting into the duodenal lumen from the medial aspect of the second part of the duodenum (Figure 5). The clinical and radiological features are similar to choledochocele, the cystic dilatation of the intraduodenal part of the distal CBD. However, duplication cyst wall shows gut signature and changes its shape on compression.

\section{Intestinal malrotation}

This occurs from rotation of the duodenum by 180 degrees anticlockwise and the colon by 90 degrees anticlockwise 

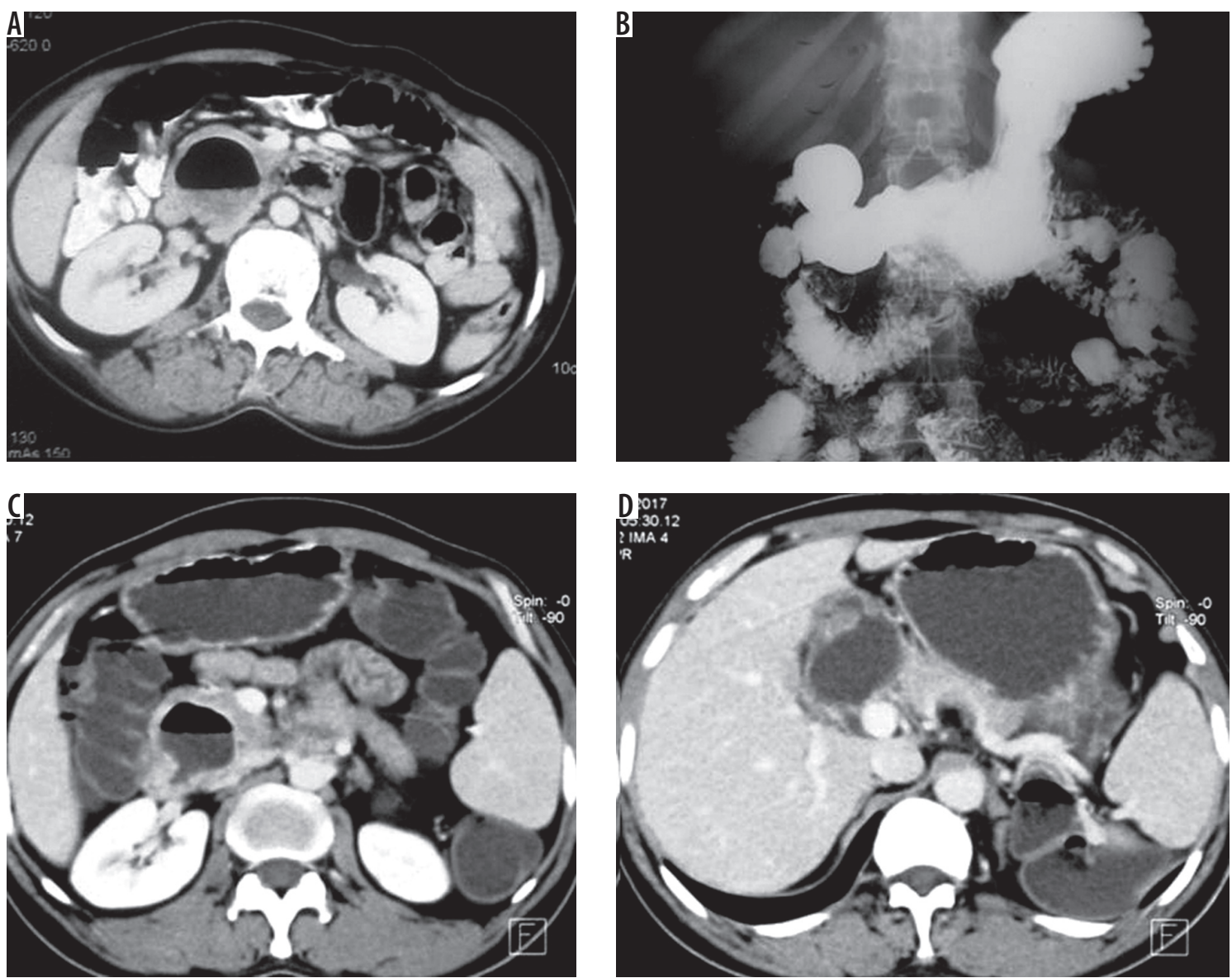

Figure 4. Axial contrast-enhanced computed tomography (CECT) image (A) of the abdomen shows a well-defined outpouching from the medial wall of the second part of the duodenum, containing air-fluid level, and causing medial displacement of head of pancreas. Upper gastrointestinal barium study in a different patient reveals smooth walled, barium-filled diverticulae from duodenum and jejunum. Axial CECT sections from the upper abdomen showing duodenal diverticulum in the second part (C), which is causing upstream central hepatic biliary radicle dilatation (D) due to compression of common bile duct, suggesting Lemmel's syndrome

due to a short mesenteric root, causing the duodenojejunal junction to lie to the right side of left-sided L2 pedicle and the caecum to lie in a slightly superior location. In malrotation, the superior mesenteric venous (SMV) lies to the left of the superior mesenteric artery (SMA) and is usually associated with midgut volvulus (Figure 6) and Ladd's band (Figure 7), causing duodenal obstruction. It must be remembered that organo-axial or mesenteroaxial gastric volvulus (Figure 8) may also cause malposition of the duodenal bulb.

\section{Infective conditions}

\section{Tuberculosis}

Duodenal tuberculosis is usually associated with gastric antral and pylorus involvement. Common radiological findings in duodenal tuberculosis are pre-bulbar or postbulbar strictures, extrinsic compression by matted lymph nodes (Figure 9), intraluminal polypoidal mass, mucosal ulceration, and complications such as perforation and fistula [9]. The radiological appearance is similar to that of Crohn's disease.
Infections predominantly affecting the proximal small bowel are Whipple's disease, giardiasis, and strongyloidiasis.

\section{Inflammatory diseases}

\section{Duodenal ulcer}

They are usually seen in the first part of the duodenum. Anterior wall ulcers are best seen on prone views. The post bulbar ulcers should raise suspicion of Crohn's disease or Zollinger-Ellison syndrome (ZES) [1]. Perforation, bleeding, and duodenal stenosis may complicate peptic ulcers.

\section{Crohn's disease}

Crohn's disease of the duodenum is very rare and accounts for $2 \%$ of cases. It is usually associated with contiguous involvement of distal stomach [10]. On barium meal examination post-bulbar mucosal ulceration, diffuse granularity, and nodularity can be seen. Mucosal abnormalities such as superficial ulcers are the earliest findings in Crohn's disease. Funnel-shaped narrowing or 

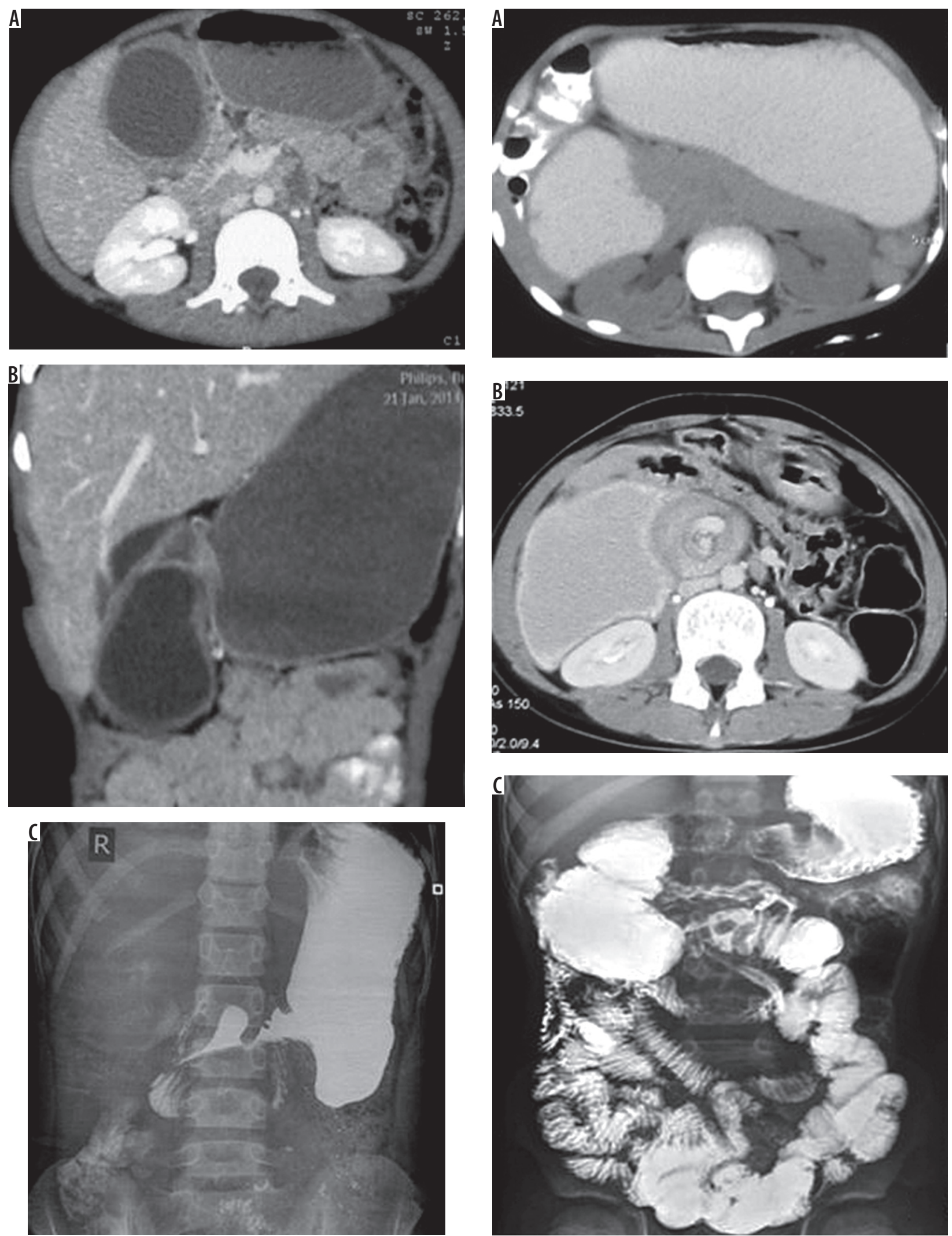

Figure 5. Axial contrast-enhanced computed tomography image (A) and coronal reconstruction (B) of upper abdomen shows a well-defined cystic structure with thick, enhancing wall, closely abutting and compressing the second part of the duodenum. On barium study $(\mathbf{C})$, a smooth, extrinsic filling defect projecting into the duodenal lumen from the medial aspect of the second part of the duodenum can be noted, suggestive of duplication cyst

Figure 6. Axial contrast-enhanced computed tomography images of abdomen revealing distended stomach and proximal duodenum (A) with abnormal relation of SMV with superior mesenteric artery and whirlpool sign (B), suggestive of volvulus. Upper gastrointestinal study (C) demonstrates right-sided small-bowel loops, left-sided large-bowel loops, and the duodenojejunal junction appears to be located at the right side of the spine, diagnosing intestinal malrotation 

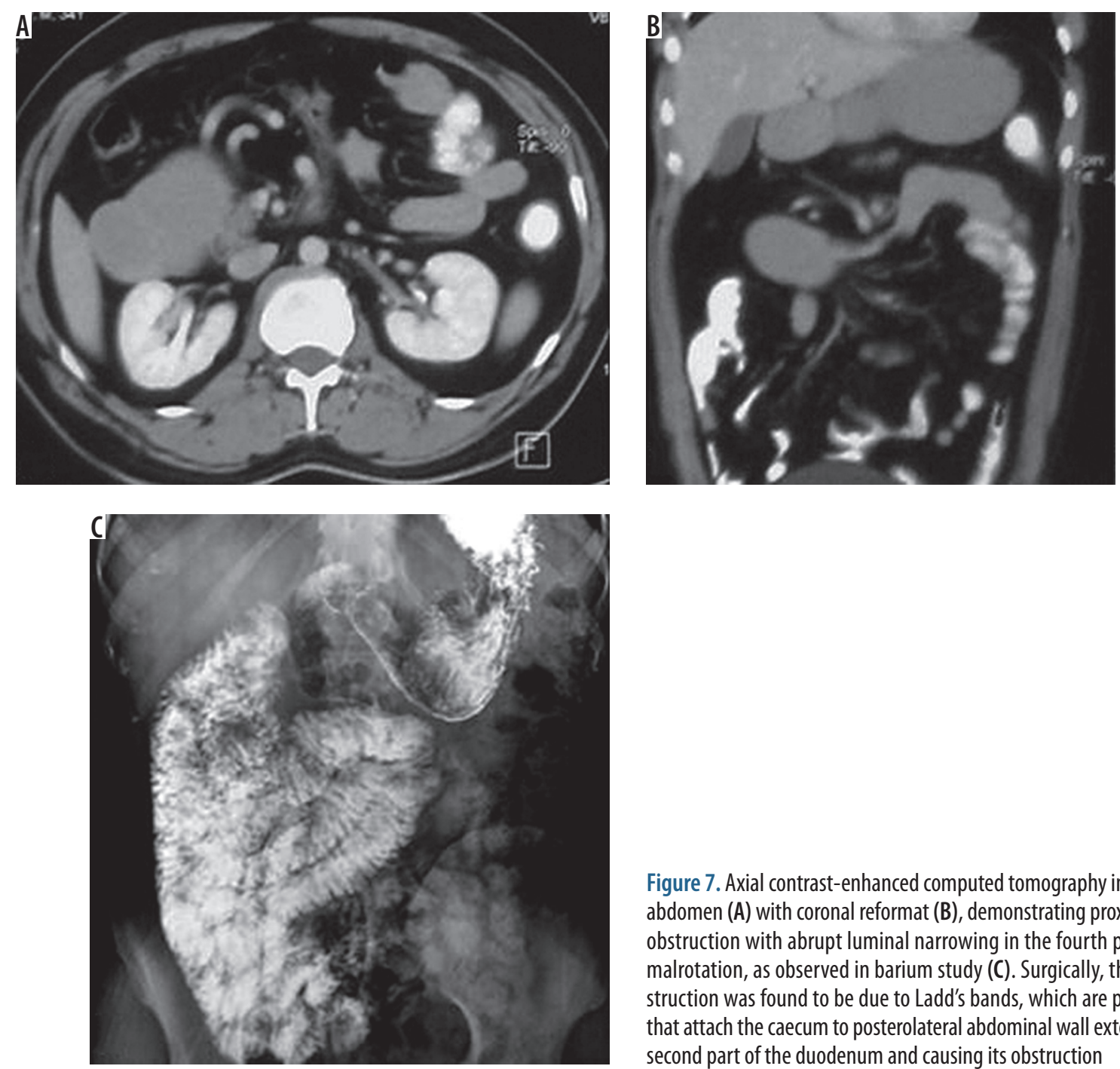

Figure 7. Axial contrast-enhanced computed tomography images of upper abdomen (A) with coronal reformat (B), demonstrating proximal duodenal obstruction with abrupt luminal narrowing in the fourth part along with malrotation, as observed in barium study (C). Surgically, the cause of obstruction was found to be due to Ladd's bands, which are peritoneal folds that attach the caecum to posterolateral abdominal wall extending into the second part of the duodenum and causing its obstruction
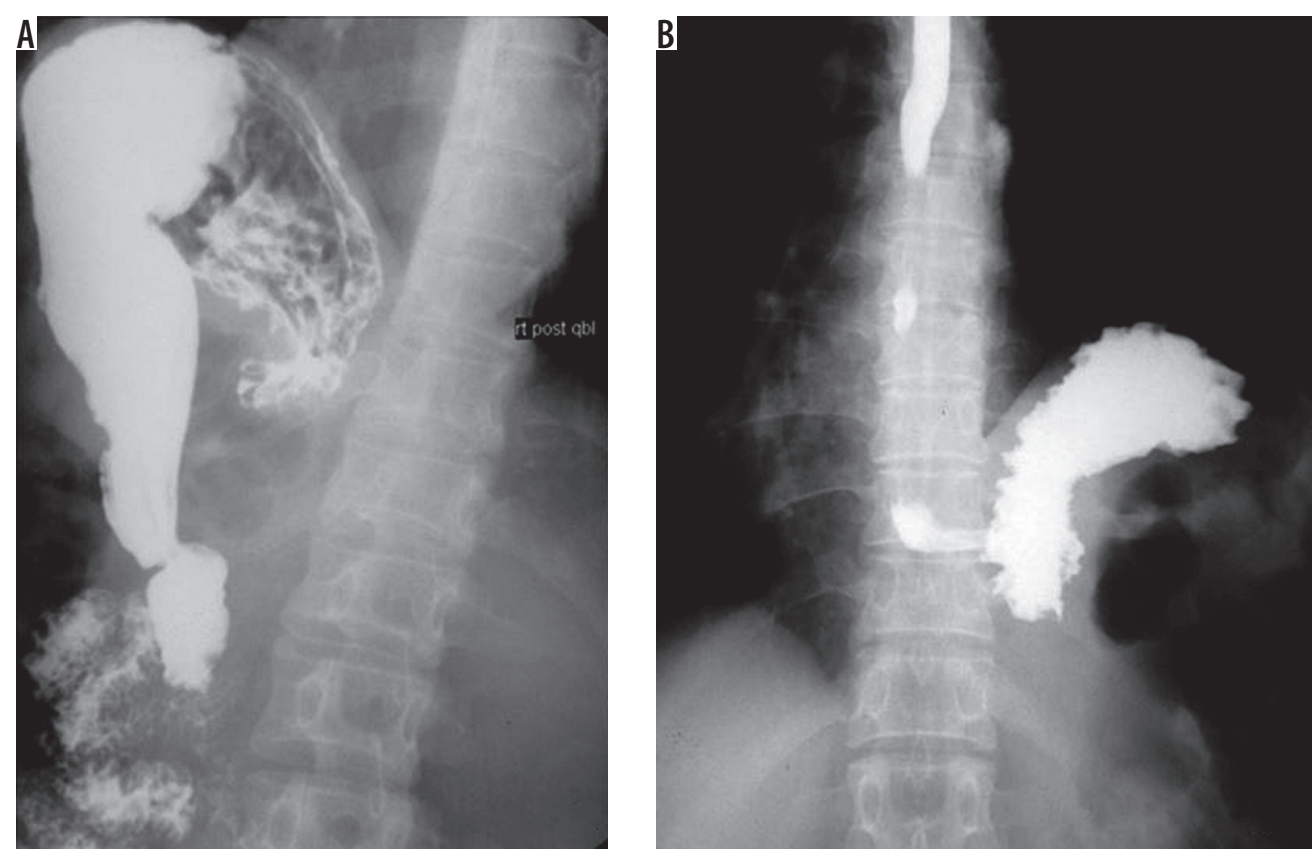

Figure 8. Barium meal (A) in an adult patient revealing organo-axial gastric volvulus causing displacement of pylorus and duodenal bulb to the left side of the spine. Barium meal in another patient (B) depicting mesentero-axial gastric volvulus 

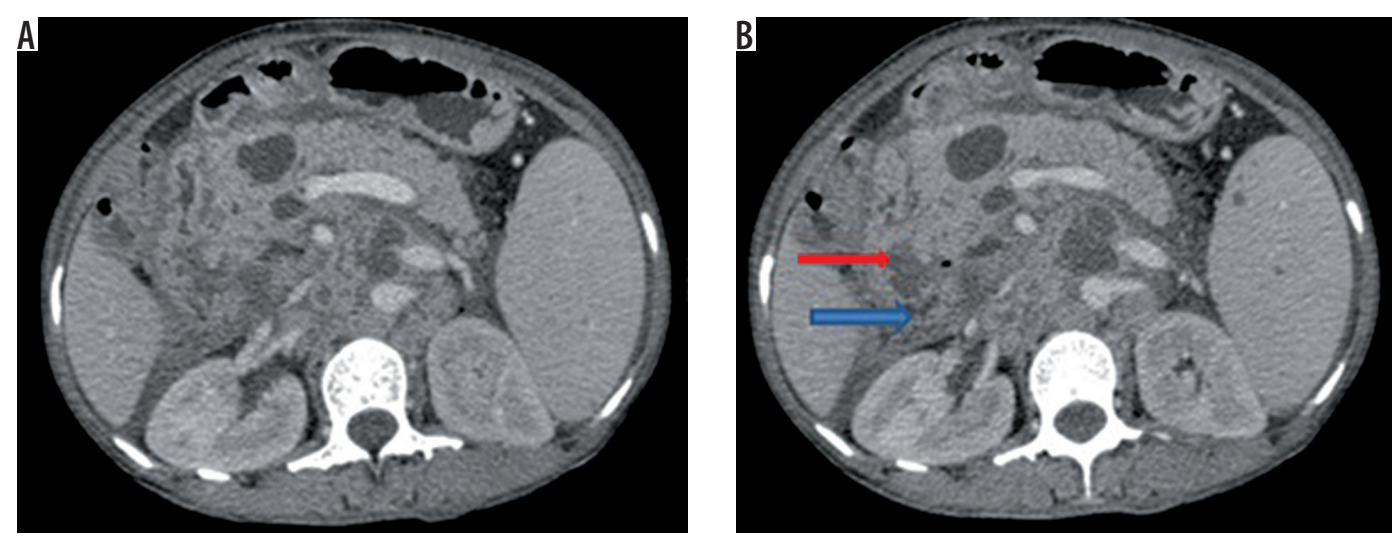

Figure 9. Axial contrast-enhanced computed tomography sections of abdomen in a known case of abdominal tuberculosis demonstrating multiple necrotic, conglomerated retroperitoneal, and peripancreatic lymph nodes (red arrow) causing extrinsic compression of duodenal lumen (blue arrow)
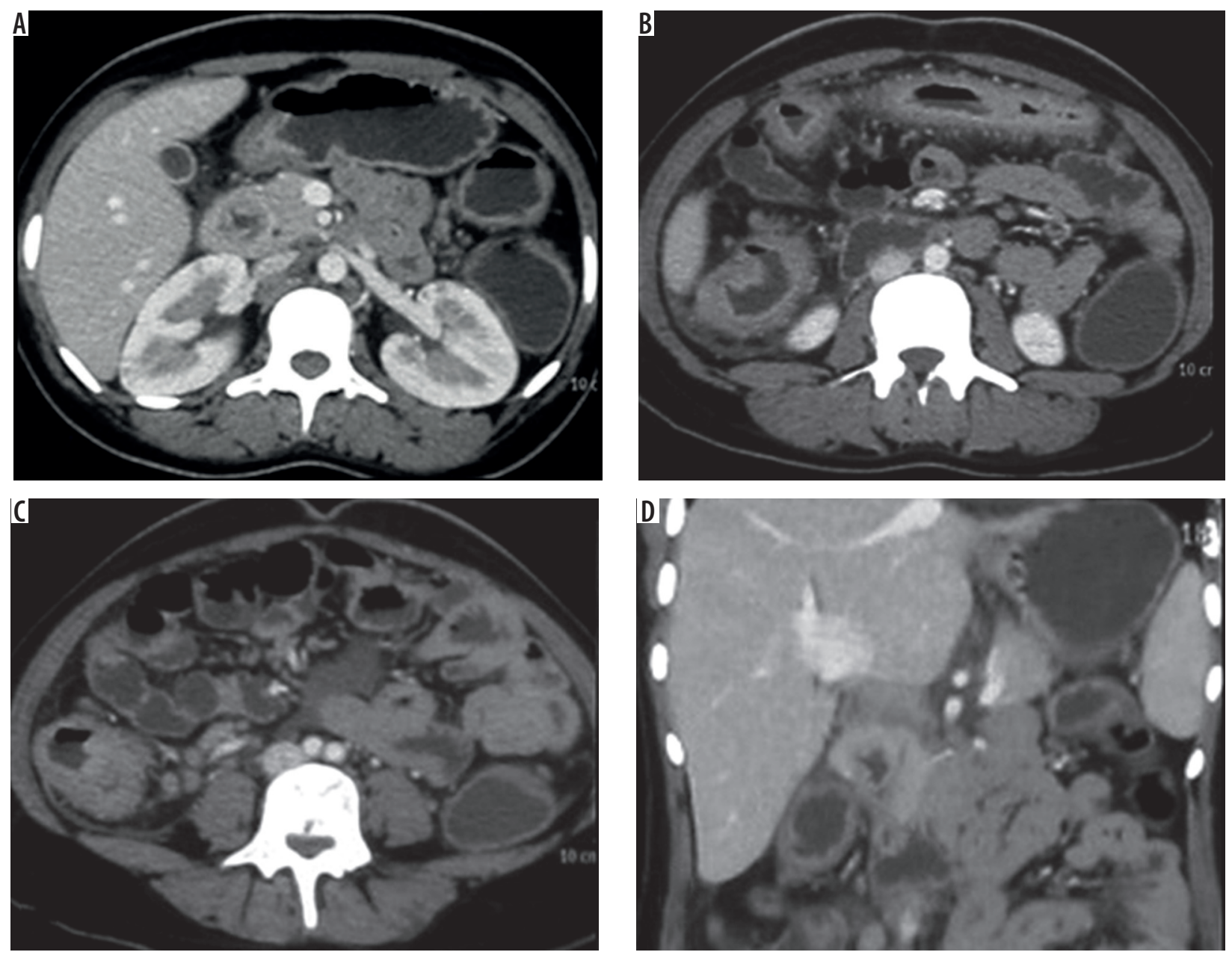

Figure 10. Axial contrast-enhanced computed tomography images of abdomen depicting multifocal short-segment concentric strictures in the duodenum (A), ascending colon (B), and ileum (C) in a case of Crohn's disease. Coronal reformat (D) depicts enhancing duodenal wall thickening with maintained stratification

stricture of the pylorus and duodenum (Figure 10) causing pseudo post-Billroth 1 gastrectomy appearance can also be seen.

\section{Paraduodenal pancreatitis}

It is a form of chronic pancreatitis focally affecting the groove between the head of pancreas, duodenum, and
CBD. CT depicts characteristic cystic thickening of the duodenal wall (Figure 11) with fibrous tissue within the pancreatico-duodenal groove showing late enhancement.

\section{Duodenal trauma}

Blunt abdominal trauma is the commonest cause and is frequently seen in children. Intramural haematoma 

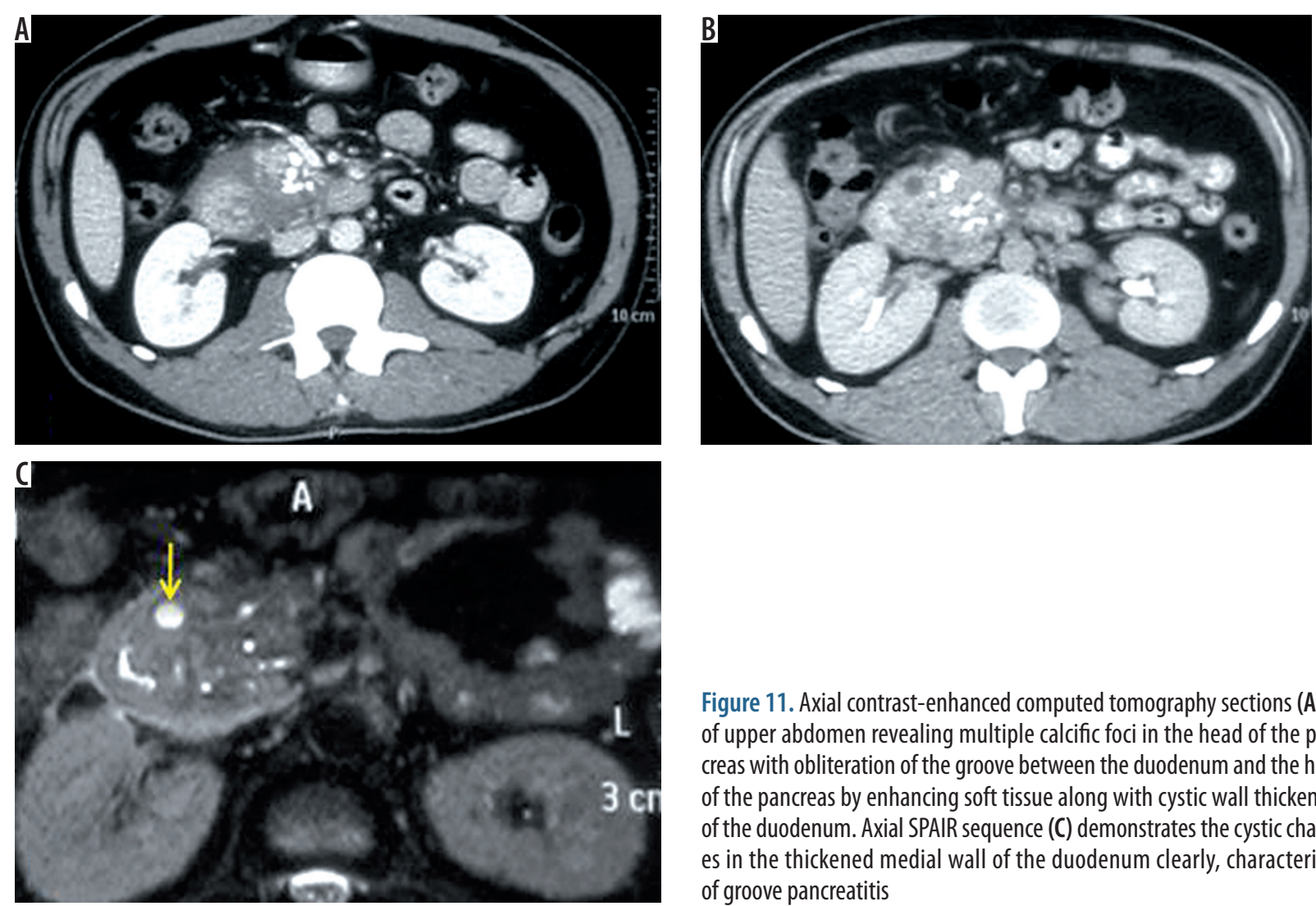

Figure 11. Axial contrast-enhanced computed tomography sections (A, B) of upper abdomen revealing multiple calcific foci in the head of the pancreas with obliteration of the groove between the duodenum and the head of the pancreas by enhancing soft tissue along with cystic wall thickening of the duodenum. Axial SPAIR sequence (C) demonstrates the cystic changes in the thickened medial wall of the duodenum clearly, characteristic of groove pancreatitis

occurs when a strong mechanical force compresses the duodenum against rigid vertebral bodies, leading to rupture of submucosal vascular plexus in the duodenum and appearing hyperdense on noncontrast-enhanced CT (NCCT) (Figure 12). The most common location of traumatic duodenal rupture is the junction of the second and third parts of the duodenum. Lack of continuity, retroperitoneal contrast leak, and pneumoretroperitoneum are the signs of duodenal perforation (Figure 13). It is crucial to identify the associated pancreatic injury. Differentiation between the intramural haematoma and perforation

is vital because perforation requires urgent surgical intervention [1].

\section{Vascular anomalies}

\section{Superior mesenteric artery syndrome}

It is characterised by constriction of the third part of the duodenum between the superior mesenteric artery and abdominal aorta due to the narrowing of the aortomesenteric angle (Figure 14). The normal aortomesenteric angle is
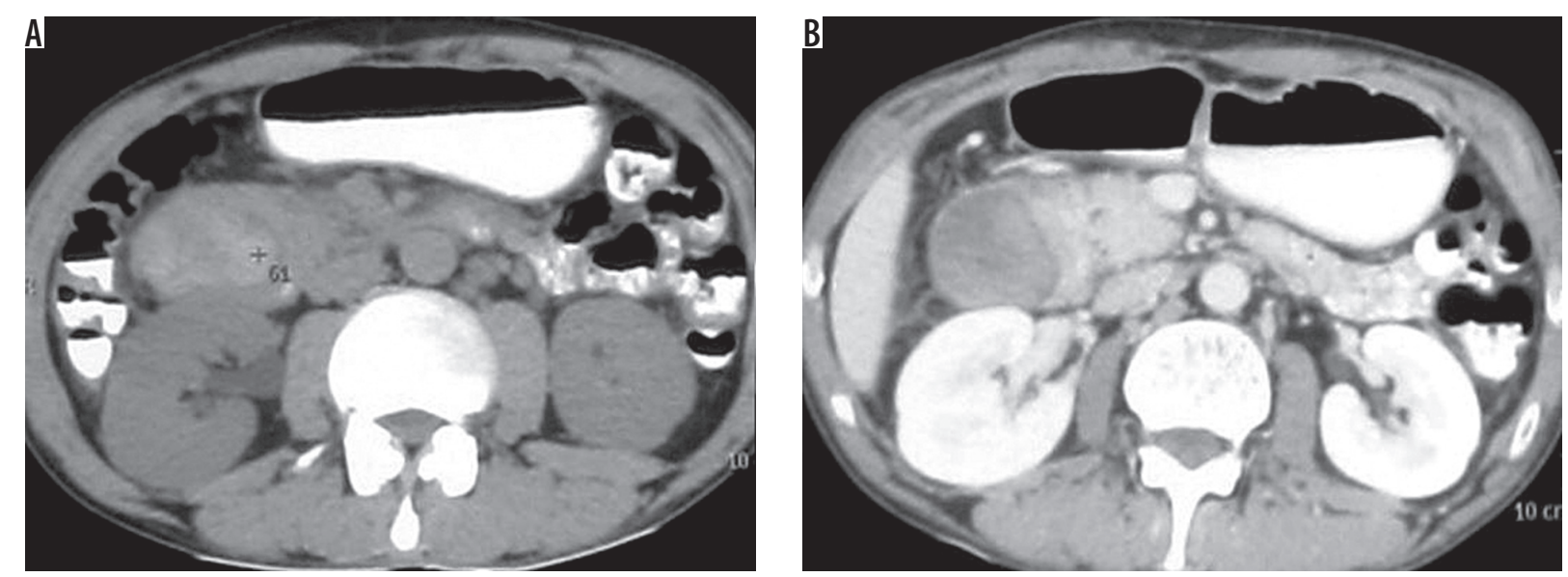

Figure 12. Axial noncontrast-enhanced computed tomography (A) section of the abdomen in a case of blunt trauma abdomen reveals hyperdense area of attenuation $61 \mathrm{HU}$ in the medial wall of the second part of the duodenum. On contrast-enhanced computed tomography sections (B), the area is nonenhancing, consistent with intramural haematoma 

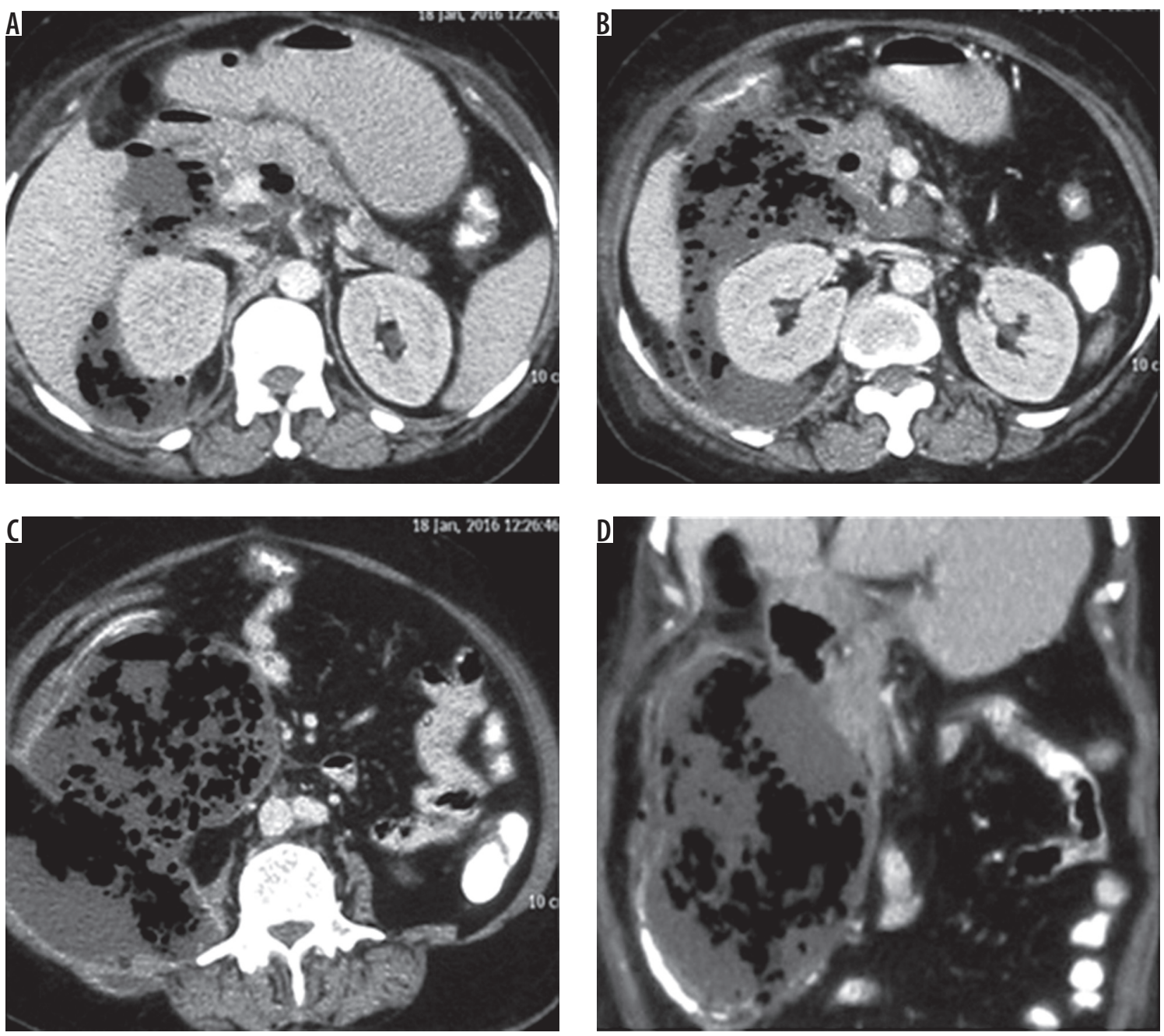

Figure 13. Axial contrast-enhanced computed tomography images $(A, B, C)$ and coronal reformats $(D)$ of the abdomen in a patient after a road traffic accident shows non-visualisation of duodenum after the bulb, along with a huge hypodense retroperitoneal collection containing air foci, suggestive of duodenal rupture

45-60 degrees, and aortomesenteric distance is 10-28 $\mathrm{mm}$ [11]. Multiple detector CT is the investigation of choice in SMA syndrome, which demonstrates decreased angle and distance in the range of 6-15 degrees and 2-8 mm. Prolonged bed rest and rapid weight loss are the risk factors for SMA syndrome.

Other vascular lesions involving duodenum are duodenal varices (seen as a solitary filling defect or thickened and serpiginous folds in the proximal duodenum), aortoduodenal fistula (seen post-abdominal aortic aneurysm repair) and preduodenal portal vein (which runs anterior to the proximal duodenum and causes proximal duodenal obstruction). Vasculitis of SMA or celiac axis and its branches supplying the duodenum can cause moderate to severe diffuse thickening of duodenal wall.

\section{Neoplasms}

\section{Benign}

The most common benign tumours of the duodenum are adenomas, leiomyomas/gastrointestinal stromal tumours (GIST), lipomas, and angiomas [12].

\section{Gastrointestinal stromal tumours}

GIST are the most common tumour of mesenchymal origin [13] with the stomach being the commonest location followed by the small intestine. In the duodenum they usually arise from the second or third parts. Abdominal pain, haemorrhage, and chronic anaemia are the clinical symptoms. It arises from the interstitial cells of Cajal, which are c-kit positive mesenchymal precursor cells. A small number of GISTs also show PDGFR- $\alpha$ mutation and DOG-1 mutation. GISTs usually arise from the subepithelial bowel wall. They may undergo ulceration, malignant degeneration, necrosis, and haemorrhage. The presence of gas in the ulcer crater helps in identifying the gut origin.

On CECT, smaller GISTs show endoluminal growth and homogenous enhancement, forming an obtuse angle with the bowel wall. GISTs can also appear as large (even greater than $30 \mathrm{~cm}$ ) well-defined, with a large necrotic centre (Figure 15). Large exophytic GIST invades the adjacent organs such as pancreas, colon, and diaphragm. Fifty per cent of the patients may develop metastasis to the liver and peritoneum [14]. 

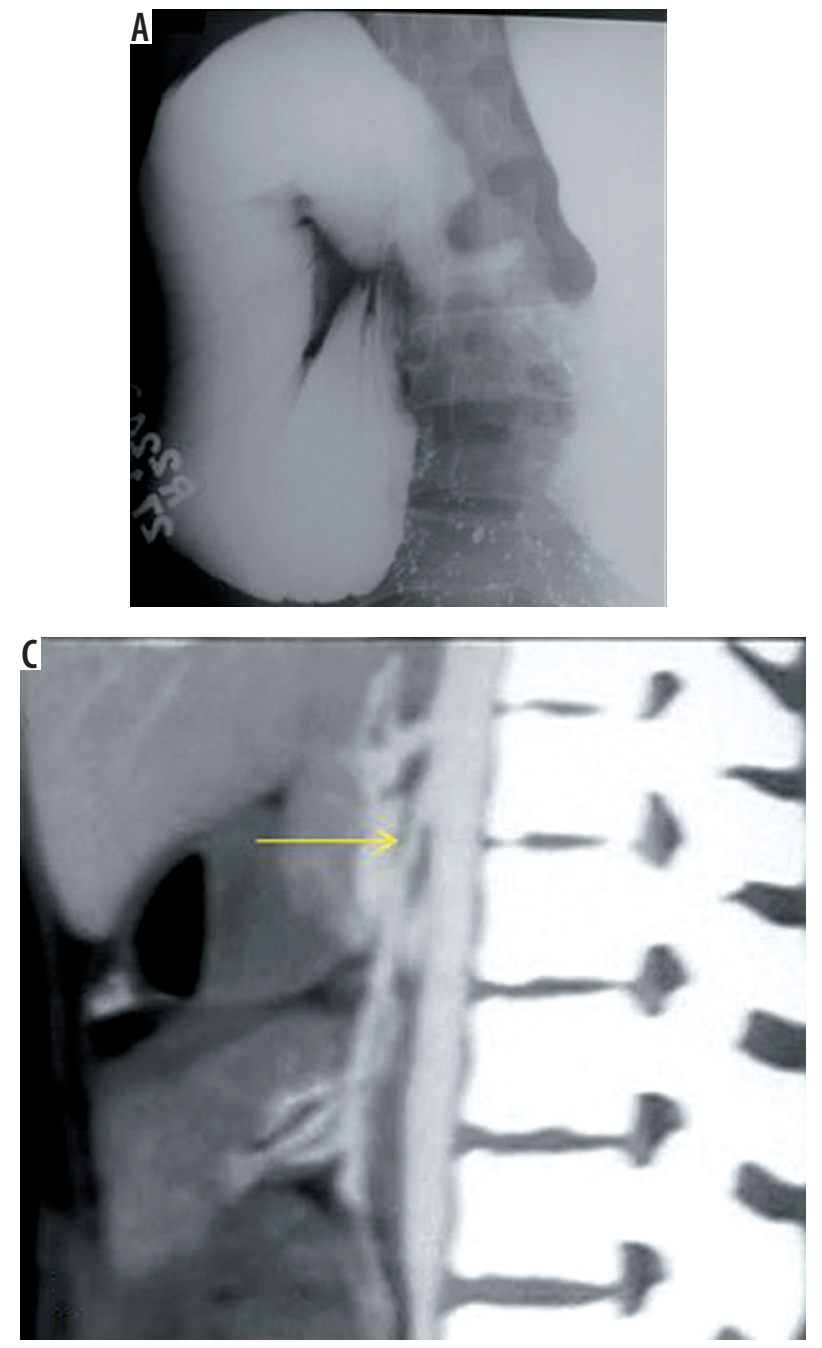

Duodenal polyps

Multiple duodenal polyps are seen in Peutz-Jeghers syndrome (PJS), Gardner syndrome, and familial adenomatous polyposis (FAP) syndrome, and they are pre-malignant. Sporadic adenomatous polyps are commonly single and sessile and are seen in the second part of the duodenum [15].

\section{Malignant}

\section{Primary adenocarcinoma}

Adenocarcinoma of the small intestine most commonly occurs in the distal duodenum and proximal jejunum, accounting for $50-70 \%$ of small bowel adenocarcinoma [16]; 50\% of small bowel adenocarcinoma occurs near the ampulla. Risk factors are Peutz-Jeghers syndrome, FAP, Lynch II syndrome, Crohn's disease, and sprue. It can present as a short-segment annular lesion, infiltrative lesion, polypoidal mass, or ulcerative lesion. Polypoidal lesions are more common. Gradual progressive luminal narrowing leads to incomplete or complete small intestinal obstruction (Figure 16). Short segment and rigid stricture with or without mucosal ulcerations and apple-core lesions can be demonstrated in barium examination. They usually pres-

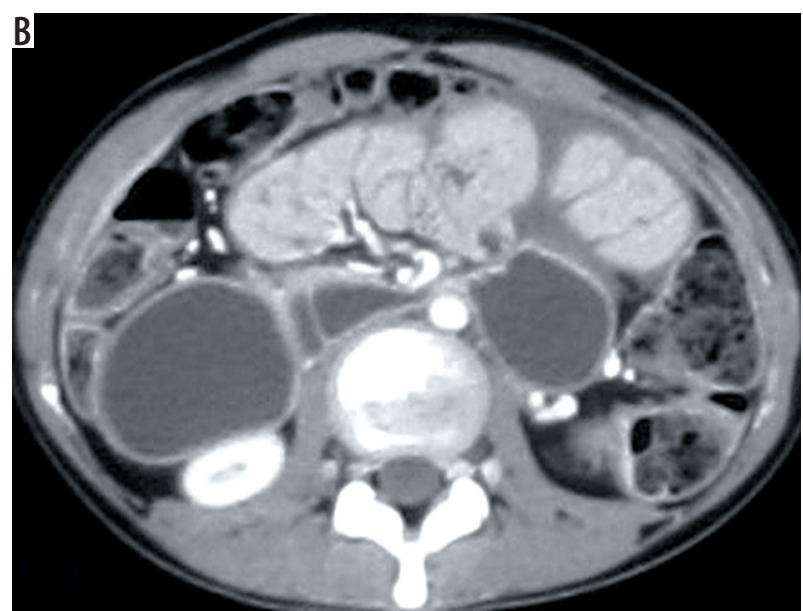

Figure 14. Upper gastrointestinal study (A) reveals overdistension of the first and second parts of the duodenum with smooth, gradual, but complete narrowing in calibre of the proximal third part of the duodenum, suggesting extrinsic compression. Axial contrast-enhanced computed tomography (CECT) (B) sections demonstrating significantly decreased aortomesenteric distance at the level where superior mesenteric artery (SMA) crosses the third part of the duodenum, with resultant proximal obstruction. Sagittal CECT (C) reformat depicting narrowed aorto-mesenteric angle (yellow arrow), consistent with SMA syndrome

ent as a heterogeneous attenuation mass with moderate contrast enhancement on CT (Figure 17) [1]. It can cause both pancreatic and biliary duct dilatation in $52 \%$ of cases. Extension to the mesentery with widespread desmoplastic reaction and hypovascular liver metastases are frequent.

\section{Malignant GIST}

Although primarily a benign lesion, malignant degeneration in GIST is not uncommon. Poor prognostic factors include distal location, size, and high mitotic activity, with the exception of oesophageal GISTs. Malignant GISTS are usually $>5 \mathrm{~cm}$ in size, unlike benign lesions, which are usually $<2 \mathrm{~cm}$. However, it is better to classify smaller tumours as having lower risk for malignancy rather than as benign. Tumour necrosis, cystic change, nuclear atypia, tumour vascularity, and degree of staining for CD117 are predictors of malignancy, although not very reliable. Collection of air in the nondependent aspect of larger cavitating tumours with necrosis is known as the “Toricelli-Bernouilli" crescentic necrosis sign. Malignant GIST mostly metastasises to liver, mesentery, and peritoneum. Uncommon features with GIST are the presence of ascites, lymphadenopathy, intestinal obstruction, and metastases in lungs [17]. 

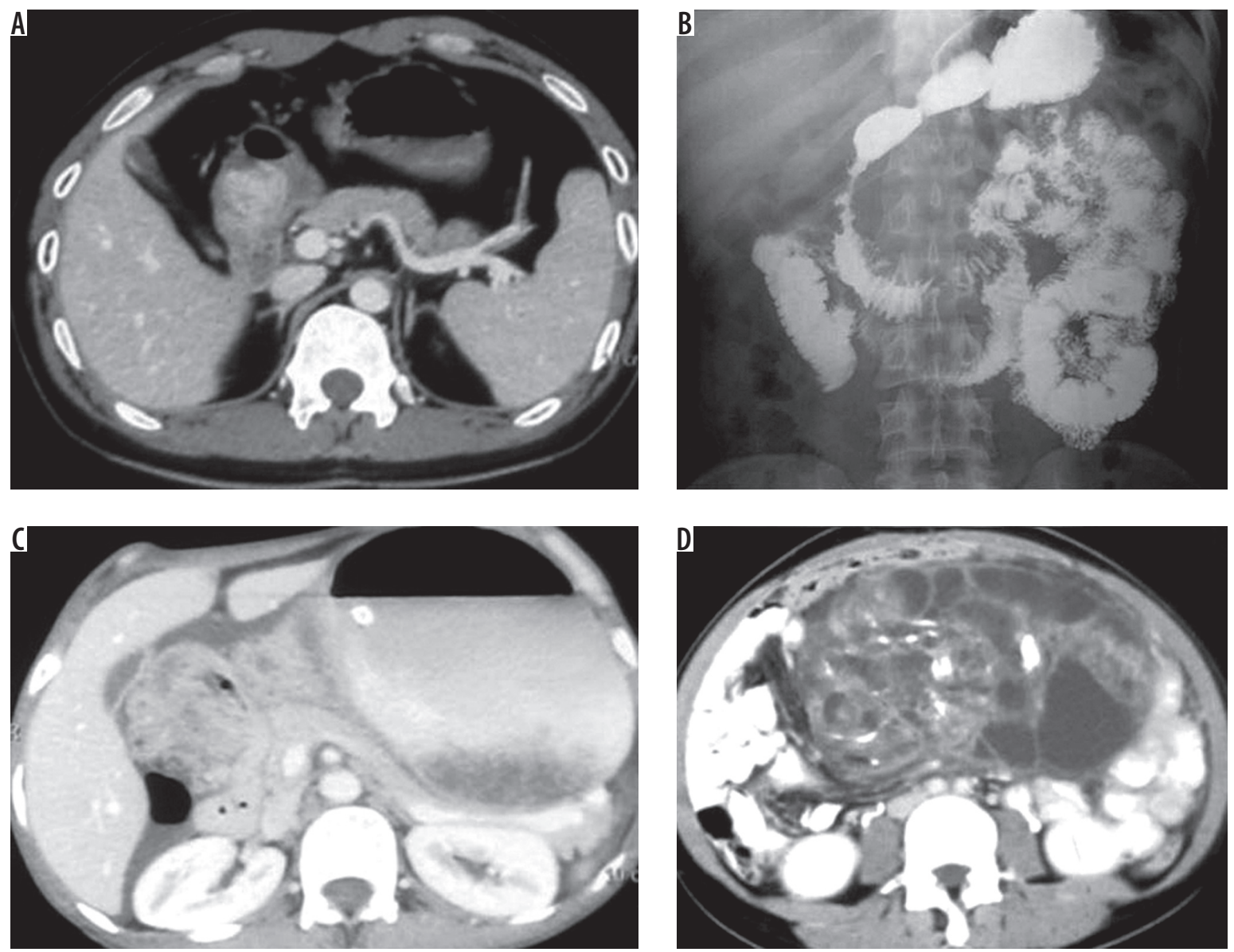

Figure 15. Axial contrast-enhanced computed tomography (CECT) sections (A) of the abdomen demonstrating a non-obstructing endoluminal growth within the bulb and second part of the duodenum, forming an obtuse angle with the duodenal wall, with heterogenous enhancement pattern, suggestive of benign submucosal lesion like gastrointestinal stromal tumours (GIST) or leiomyoma. Upper gastrointestinal study in another patient reveals widening of the C-loop of the duodenum (B), with mucosal irregularities and ulcerations in the second part of the duodenum, suggestive of lesion with endoexoenteric component. Axial CECT sections (C, D) in the same patient depict a large, well-defined, heterogeneously enhancing mass lesion with areas of calcification and necrosis within noted closely abutting, compressing and widening of the Cloop of the duodenum. However, no obvious oral contrast or air density was noted within the lesion. A biopsy from the lesion showed c-kit-positive mesenchymal cells consistent with diagnosis of GIST

\section{Duodenal neuroendocrine tumours}

Duodenal neuroendocrine tumours (D-NET) are rare and may be gastrinomas, somatostatinomas, non-functional serotonin containing tumours, poorly differentiated neuroendocrine carcinomas, and gangliocytic paragangliomas. The most common location of D-NET is the first or second duodenal segments. Twenty per cent of these tumours appear in the periampullary region [18] and can cause biliary obstruction, obstructive jaundice, and pancreatitis. Multiple tumours are seen in MEN-1 and ZES. D-NETs usually present as intraluminal polypoid lesion submucosal masses with or without mucosal ulceration. They characteristically show early arterial enhancement and delayed washout on cross-sectional imaging.

\section{Lymphoma}

Secondary lymphoma of the duodenum is commoner than primary lymphoma. They may present as nodular or polypoidal masses or ulcerative lesions or infiltrating tumour masses (Figure 18). Primary intestinal lymphoma is usu- ally NHL and accounts for 20\% of cases [19]. Diffuse large B-cell lymphoma (DLBCL) and extranodal marginal-zone lymphoma (associated with Helicobacter pylori infection) are the common types of B-cell lymphoma. Enteropathytype intestinal $\mathrm{T}$ cell lymphoma is a peripheral T-cell lymphoma that often involves the small intestine [20].

\section{Secondary malignant involvement of duodenum}

Malignant melanoma, lung (squamous cell variant), breast (lobular carcinoma), and thyroid cancers are common primaries [21] that metastasize to the duodenum. It can present as multiple submucosal nodules, intraluminal polypoidal masses, and diffuse wall thickening. Multiple submucosal nodules with central ulceration produce characteristic bull's eye or target appearance on barium examination. Stomach carcinoma and lymphoma can directly extend into the duodenum across the pylorus. Pancreatic head adenocarcinoma can cause enlargement of the duodenal loop or involve the duodenum. Carcinoma of jejunum (Figure 19) and colon can also involve the duodenum with the formation of coloduodenal fistula. 

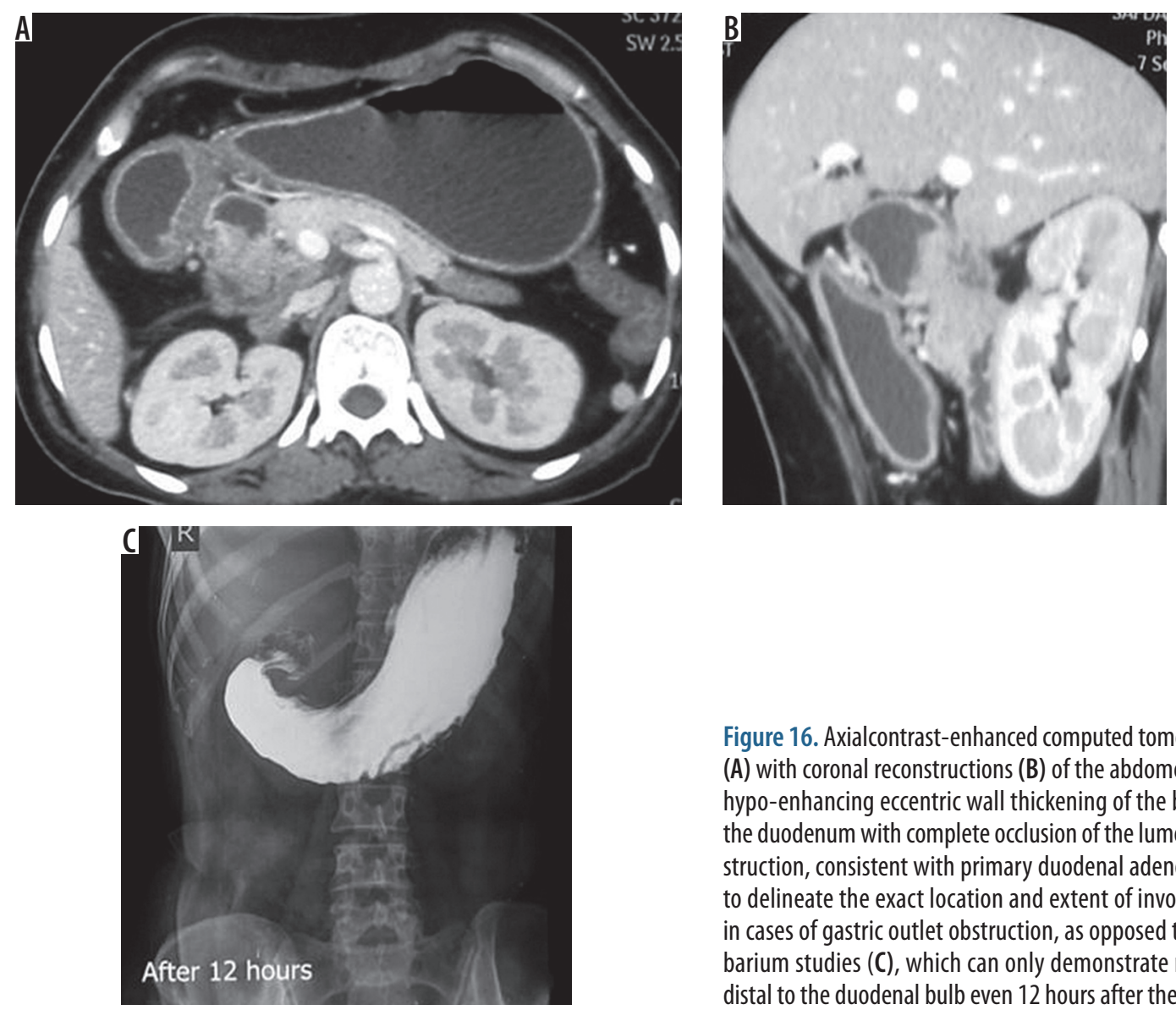

Figure 16. Axialcontrast-enhanced computed tomography (CECT) sections (A) with coronal reconstructions (B) of the abdomen show homogenously hypo-enhancing eccentric wall thickening of the bulb and second part of the duodenum with complete occlusion of the lumen causing proximal obstruction, consistent with primary duodenal adenocarcinoma. CECT helps to delineate the exact location and extent of involvement of malignancy in cases of gastric outlet obstruction, as opposed to conventional luminal barium studies $(\mathbf{C})$, which can only demonstrate non-passage of barium distal to the duodenal bulb even 12 hours after the start of study

\section{Post-surgical changes in the duodenum}

CT vividly depicts the majority of postoperative complications like wound dehiscence, haematoma, anastomotic leakage, hernia, bowel obstruction, and infection [1]. Early gastric carcinoma is treated with resection followed by gastrojejunostomy. The part of the duodenum draining the biliary and pancreatic systems forms the afferent limb of gastrojejunostomy. Ulceration or recurrent carcinoma of the gastrojejunostomy anastomosis leads to afferent limb obstruction with resultant biliary and pancreatic duct dilatation. Even when the tumour cannot be imaged, a dilated afferent limb is suspicious for tumour recurrence [1].

\section{Radiological approach to duodenal pathologies}

Is duodenal obstruction present? If present: severity, transition point, aetiology, and complications of obstruction may be assessed with CECT or barium meal followthrough. Causes of obstruction could be congenital, vascular, or neoplastic.

Is bowel wall thickening present? If present: the degree and symmetry of thickening is assessed. Mild thickening (3-4 mm) is seen in Crohn's disease or infection, whereas moderate $(5-9 \mathrm{~mm})$ to severe $(>10 \mathrm{~mm})$ thickening is seen in Crohn's disease, vasculitis, lymphoma, and adenocarcinoma. Asymmetric thickening is seen in neoplastic

aetiology, while symmetric thickening may suggest a benign aetiology like Crohn's disease and tuberculosis or a malignant pathology like lymphoma.

Is the duodenal involvement focal or diffuse? Focal involvement of $<2$ duodenal segments may indicate neoplastic or inflammatory aetiology, while diffuse involvement of all segments is seen in vasculitis or ischaemia.

What is the mural enhancement pattern? Four common enhancement patterns are commonly observed as follows:

- target/mural stratification: in Crohn's disease and infection,

- homogenous: lymphoma, intramural haemorrhage,

- heterogenous: malignancy, GIST, metastasis,

- decreased/ absent: ischaemia.

Associated abnormality in retroperitoneum and vessels: involvement of adjacent structures, vessels, and retroperitoneal lymphadenopathy may give a clue regarding the aetiology.

\section{Recent advances in imaging of the duodenum}

Many new imaging techniques giving high-resolution anatomical and functional information regarding the duodenum and its pathologies have recently surfaced. Perfusion CT enables an analysis of angiogenesis and tumour enhancement. MRI fluoroscopy allows real-time visualisation of bowel movement, detects abnormalities 

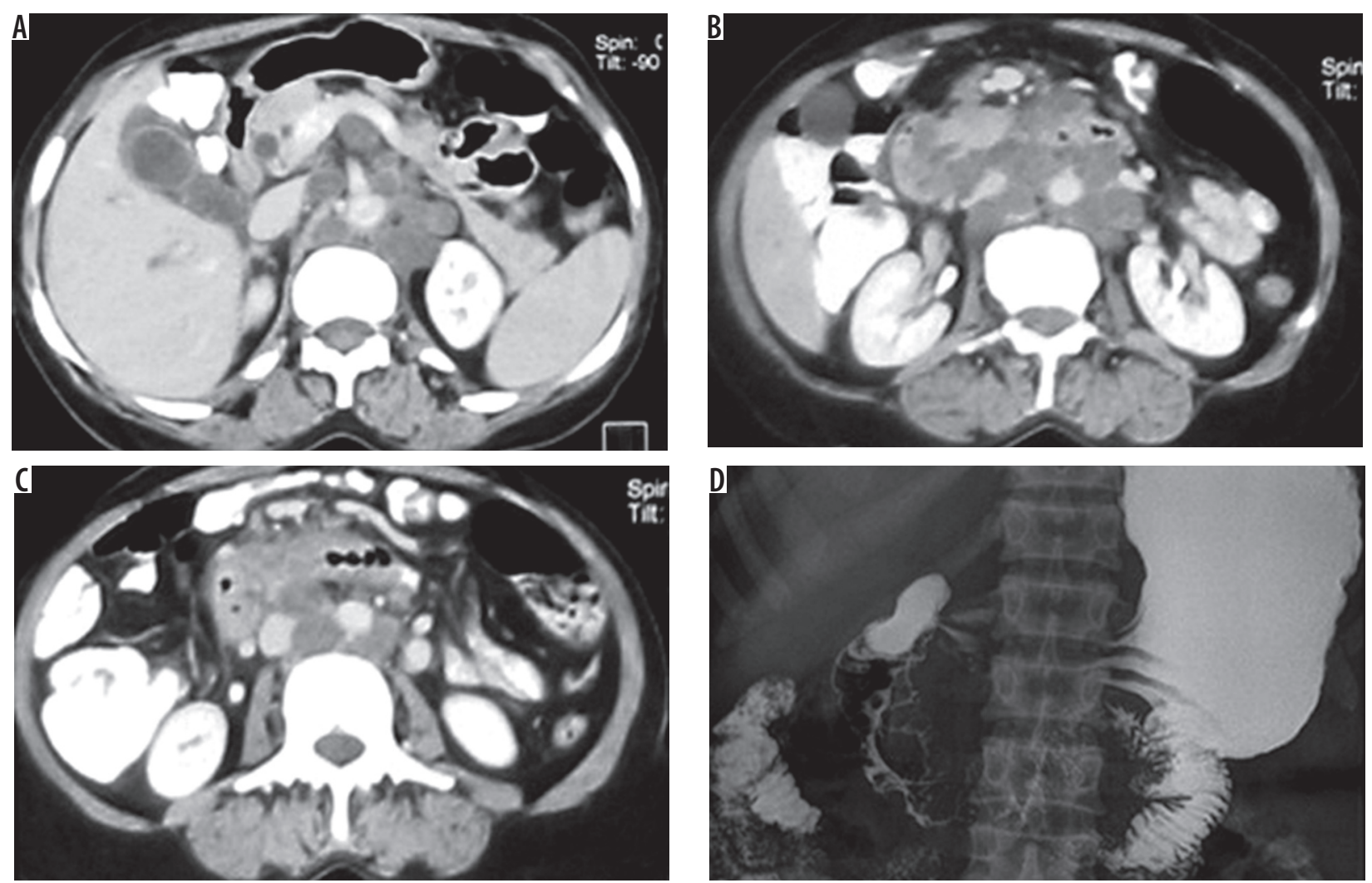

Figure 17. Axial contrast-enhanced computed tomography (A, B, C) images of upper abdomen shows eccentric hypo-enhancing wall thickening along the medial aspect of the second, third, and fourth parts of the duodenum along with multiple necrotic nodes along the celiac artery and in the retroperitoneum. On upper gastrointestinal study (D) in the same patient, definite mucosal irregularity with luminal narrowing was noted in corresponding areas, suggesting primary adenocarcinoma of the duodenum
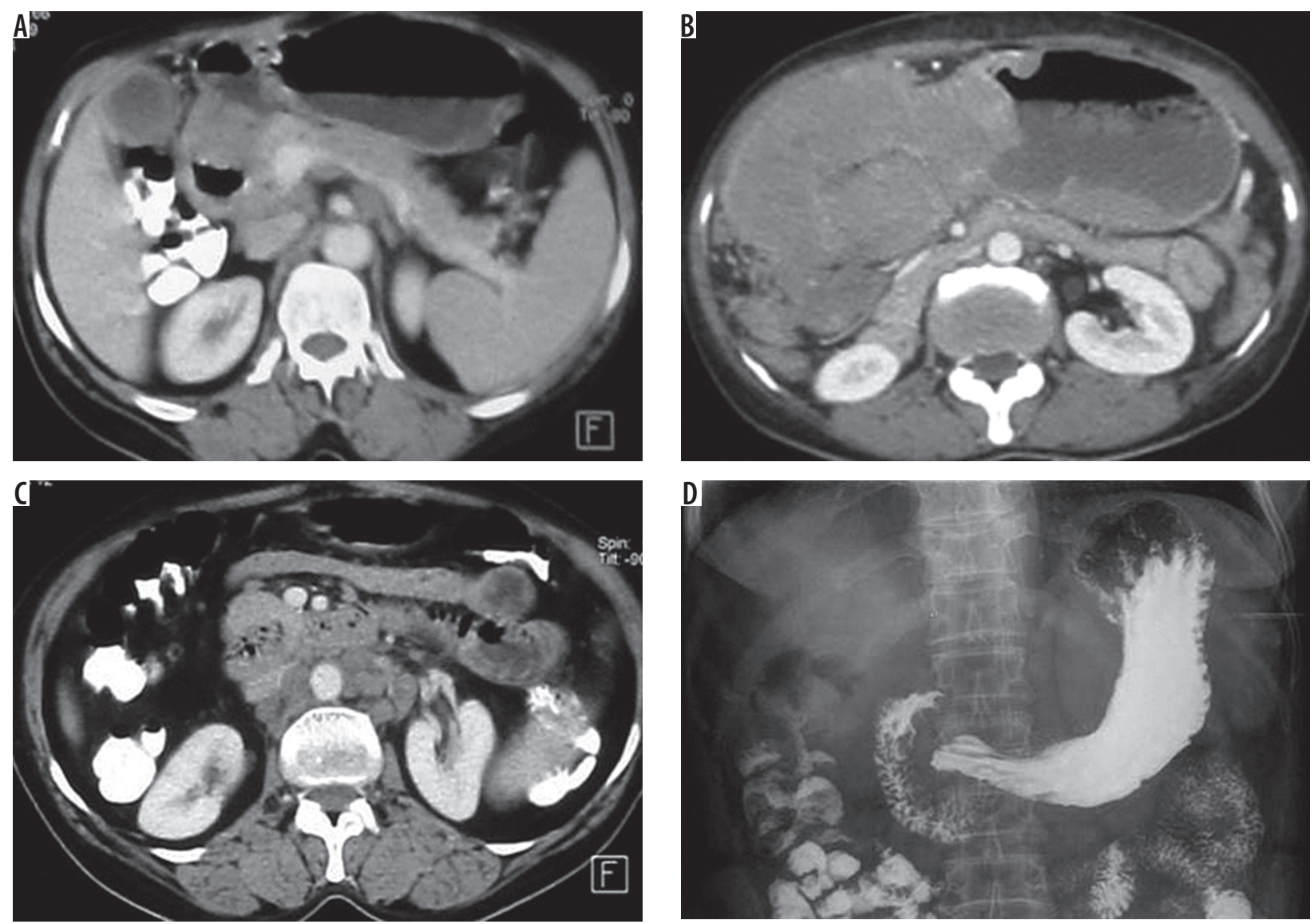

Figure 18. Axial contrast-enhanced computed tomography (CECT) (A, B) sections of upper abdomen in a known case of non-Hodgkin's lymphoma reveals smooth, homogenously hypoenhancing concentric wall thickening of the duodenum with contiguous involvement of the pylorus. CECT in another patient with gastro-duodenal lymphoma shows multiple retroperitoneal, hypoenhancing, discrete nodes (C). Barium study in another case of duodenal lymphoma depicts classic absence of proximal obstruction and maintained mucosal pattern of duodenum (D) 


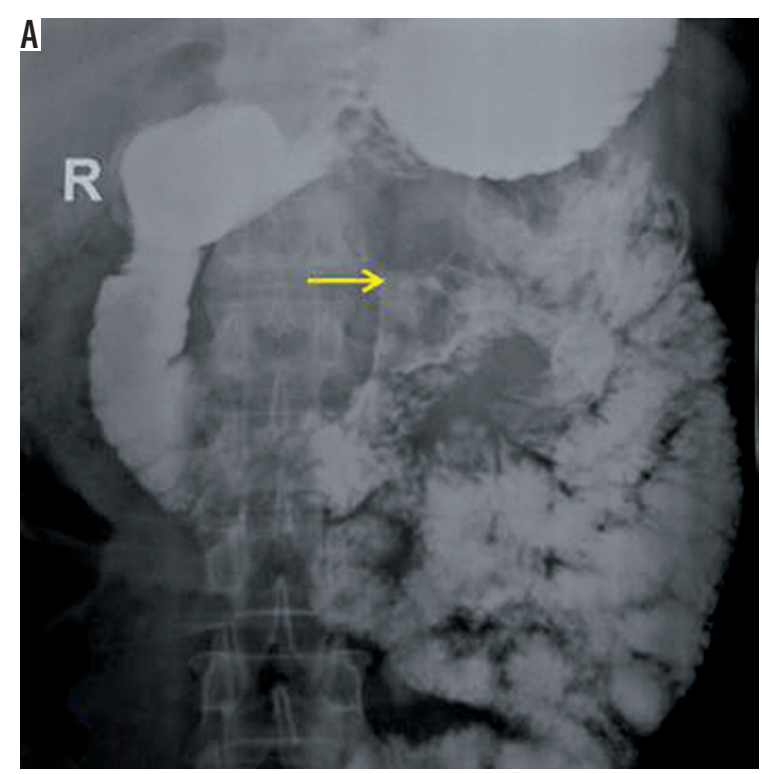

in peristalsis, and helps differentiate functional bowel spasm from fibrotic strictures. DW-MRI, which has a proven role in the detection of malignant duodenal lesions and nodal metastasis, is now also being utilised in the assessment of activity in inflammatory bowel disease [22]. Contrast-enhanced ultrasound is another recent tool for dynamic assessment of vascularity in duodenal masses and inflammation [21]. Wire-guided intraduodenal ultrasound is also being tried in the evaluation of enlarged ampullary lesions [23].

\section{Conclusions}

Computed tomography provides excellent anatomic detail and exhibits high diagnostic specificity in recognition of

\section{References}

1. Jayaraman MV, Mayo-Smith WW, Movson JS, et al. CT of the duodenum: an overlooked segment gets its due. Radiographics 2001; 21: $147-160$

2. Choudhry MS, Rahman N, Boyd P, Lakhoo K. Duodenal atresia: associated anomalies, prenatal diagnosis and outcome. Pediatr Surg Int 2009; 25: 727-730.

3. Chiarenza SF, Bucci V, Conighi ML, et al. Duodenal atresia: open versus MIS repair - analysis of our experience over the last 12 years. Biomed Res Int 2017: 4585360.

4. Sandrasegaran K, Patel A, Fogel EL, et al. Annular pancreas in adults. Am J Roentgenol 2009; 193: 455-460.

5. Lath CO, Agrawal DS, Timins ME, Wein MM. Portal annular pancreas: the pancreatic duct ring sign on MRCP. Radiol Case Rep 2015; 10: 42-45.

6. Zyromski NJ, Sandoval JA, Pitt HA, et al. Annular pancreas: dramatic differences between children and adults. J Am Coll Surg 2008; 206: 1019-1025.

7. Liu R, Adler DG. Duplication cysts: diagnosis, management, and the role of endoscopic ultrasound. Endosc Ultrasound 2014; 3: 152-160.

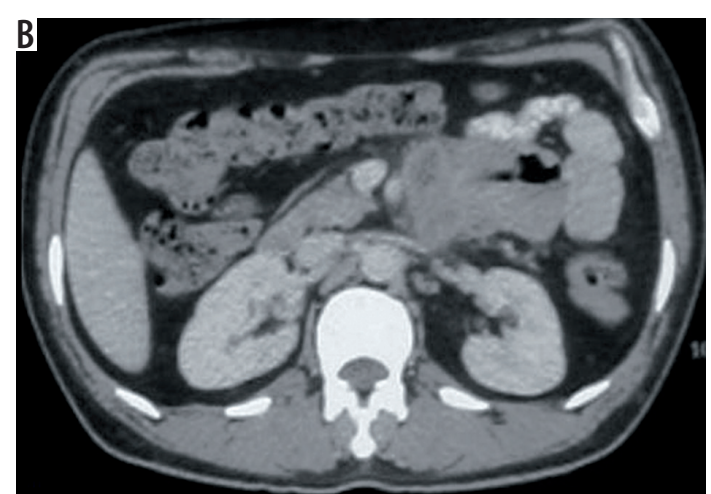

Figure 19. A, B) Upper-gastrointestinal study (A) in a patient with obstruction shows focal filling defect with mucosal irregularity in the distal third and fourth parts of the duodenum and proximal jejunum (yellow arrow) along with mild proximal obstruction. On contrast-enhanced computed tomography abdomen (B), hypoenhancing thickening of jejunum with contiguous involvement of distal duodenum was noted, suggestive of infiltration by jejunal adenocarcinoma

duodenal pathologies because it permits direct imaging of the intestinal wall as well as secondary abnormal changes in mesentery and adjacent structures. Primary duodenal malignancies and secondary involvement by adjacent malignancies can be easily and reliably detected by CT. Besides being the mainstay of post-operative imaging, CT also plays a crucial role in the diagnosis of traumatic duodenal injury by differentiating between mural haematoma and a duodenal perforation because the latter requires immediate surgical intervention.

\section{Conflict of interest}

The authors report no conflict of interest.

8. Chen JJ, Lee HC, Yeung CY, et al. Meta-analysis: the clinical features of the duodenal duplication cyst. J Pediatr Surg 2010; 45: 1598-1606.

9. Kshirsagar AY, Kanetkar SR, Langade YB, et al. Duodenal stenosis secondary to tuberculosis. Int Surg 2008; 93: 265-267.

10. Nugent FW, Richmond M, Park SK. Crohn's disease of the duodenum. Gut 1977; 18: 115-120.

11. Gustafsson L, Falk A, Lukest PJ, Gamklou R. Diagnosis and treatment of superior mesenteric artery syndrome. Br J Surg 1984; 71: 499-501.

12. De Bakker BS, Phoa SSKS, Kara M, et al. The vanishing duodenal polyp: mesenteric invagination presenting as duodenal pseudopolyp. BMJ Case Rep 2017: bcr-2016-214998.

13. He Z, Sun C, Zheng Z, et al. Endoscopic submucosal dissection of large gastrointestinal stromal tumors in the esophagus and stomach. J Gastroenterol Hepatol 2013; 28: 262-267.

14. Kang HC, Menias CO, Gaballah AH, et al. Beyond the GIST: mesenchymal tumors of the stomach. Radiographics 2013; 33: 1673-1690.

15. Culver EL, McIntyre AS. Sporadic duodenal polyps: classification, investigation, and management. Endoscopy 2011; 43: 144-155. 
16. Buckley JA, Fishman EK. CT evaluation of small bowel neoplasms: spectrum of disease. Radiographics 1998; 18: 379-392.

17. Sureka B, Mittal MK, Sinha M, Thukral BB. Imaging spectrum of gastrointestinal stromal tumor. Indian J Med Paediatr Oncol 2014; 35: 143-148.

18. Sato Y, Hashimoto S, Mizuno KI, et al. Management of gastric and duodenal neuroendocrine tumors. World J Gastroenterol 2016; 22: 6817-6828.

19. Ghai S, Pattison J, Ghai S, et al. Primary gastrointestinal lymphoma: spectrum of imaging findings with pathologic correlation. Radiographics 2007; 27: 1371-1388.

20. Lewis RB, Mehrotra AK, Rodríguez P, et al. From the radiologic pathology archives: gastrointestinal lymphoma: radiologic and pathologic findings. Radiographics 2014; 34: 1934-1953.
21. Washington K, McDonagh D. Secondary tumors of the gastrointestinal tract: surgical pathologic findings and comparison with autopsy survey. Mod Pathol 1995; 8: 427-433.

22. Sinha R. Recent advances in intestinal imaging. Indian J Radiol Imaging 2011; 21: 170-175.

23. Kim SH, Moon JH, Lee YN, et al. Wire-guided intraduodenal ultrasonography using a catheter probe in the differential diagnosis of enlarged ampullary lesions. J Gastroenterol Hepatol 2017; 32: 278-282. 